\title{
Branching Ratio in the Decay of Polonium-210
}

\author{
R. W. Hayward, D. D. Hoppes, and W. B. Mann
}

\begin{abstract}
Using a micro-calorimeter to determine the alpha-particle activity and a sodium-iodide scintillation counter of high efficiency to measure the gamma-ray intensity, the branching ratio of polonium- 210 has been found to be equal to $(1.22 \pm 0.06) \times 10^{-5}$. This value is based on the assumption that the energy of the main alpha-particle group from polonium-210 is $5.301 \mathrm{Mev}$. In the calibration of the scintillation counter the angular anisotropy of the gamma rays from cobalt-60 was found to be $1.164 \pm 0.002$.
\end{abstract}

\section{Introduction}

The number of gamma quanta emitted per disintegration of polonium-210 was recently determined by Grace, Allen, West, and Halban [1] ${ }^{1}$ in the course of the investigation of a method for standardizing polonium sources by measurement of the hard $0.804-$ Mev [2: gamma radiation. The intensity of the alpha radiation emitted from a $200-\mathrm{mc}$ source of polonium-210 deposited on one side of a platinum foil was measured directly by means of a low-geometry defined-solid-angle method, whereas the $0.804-\mathrm{Mev}$ gamma-ray intensity was determined in terms of a source of cobalt-60 by means of a cylindrical aluminum Geiger-Müller counter of unspecified filling. The absolute disintegration rate of the cobalt-60 source, which it is assumed was of a similar geometry, to that of polonium-210, was determined by means of the method of beta-gamma coincidence counting whereas the relative efficiencies of the Geiger-Müller counter to the gamma radiations from cobalt-60 and polonium-210 were assumed to be the same as those determined for a counter of similar geometry by Bradt, Gugelot, Huber, Medicus, Preiswerk, and Scherrer [3].

The opportunity has arisen to repeat the measurement of the branching ratio of polonium-210 using, however, a micro-calorimetric method to determine the rate of alpha-particle emission and a sodiumiodide scintillation counter, of high detection efficiency, to determine the intensity of the $0.804-\mathrm{Mev}$ gamma rays by comparison with a cobalt-60 source, the gamma-ray intensity of which was determined by the method of gamma-gamma coincidence counting.

\section{Preparation of the Polonium-210 Sources}

Experiments were performed on two polonium-210 sources, the first of which, however, was too weak to give satisfactory accuracy. This had been supplied sealed in a nickel tube about $1.5-\mathrm{cm}$ long and 0.15 $\mathrm{cm}$ in external diameter. The gamma-ray spectrum indicated some energetic gamma-emitting contamination, but with an activity corresponding to $3.7 \mathrm{mc}$ only a very approximate value for the branching ratio of $1.1 \times 10^{-5}$ gamma quantum per alpha particle was obtained.

\footnotetext{
1 Figures in brackets indicate the literature references at the end of this paper.
}

The second polonium-210 source was prepared from some $60 \mathrm{mc}$ of solution that had been carefully purified prior to delivery. This purification consisted of depositing polonium-210 onto a silver foil from a stock solution containing approximately $130 \mathrm{mc}$ of polonium-210. The silver foil was then washed free from solution and dissolved in concentrated nitric acid. After expelling most of the nitric acid by evaporation the silver was precipitated by means of hydrochloric acid. The solution was filtered from the silver chloride and its acidity adjusted for deposition on a second silver foil, which was again processed in the same way as the first, the filtrate from the silver chloride being the final solution containing some $60 \mathrm{mc}$ of purified polonium-210.

Upon receipt of this solution its acidity was adjusted to a value of $\mathrm{pH}$ equal to 1 and $0.1 \mathrm{~g}$ of finely powdered silver was allowed to stand in the solution overnight. After standing for this time the solution was filtered through a plug of glass wool in a glass tube having external and internal diameters of approximately 3 and $2 \mathrm{~mm}$. The silver powder that was retained by the glass-wool plug was ultimately found to have extracted some $50 \mathrm{mc}$ of the polonium-210. The glass tube was then fused off below the glass-wool plug and above the silver powder to give a source $37-\mathrm{mm}$ long and whose approximate form is illustrated in figure 1 . On examination of its gamma-ray spectrum, this source was found to be free from the contamination that had been present in the first source.

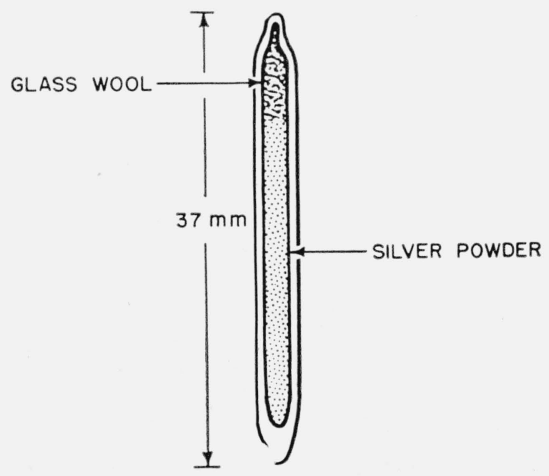

Figure 1. Polonium-210 source. 


\section{Measurement of the Alpha-Particle Activity}

The alpha-particle intensity of the polonium-210 source was determined by means of a gold-cup radiation balance that was constructed to compare four national radium standards [4]. The energy emission was combined with an average value ${ }^{2}$ of $5.301 \mathrm{Mev}$ for the energy of the polonium-210 alpha particles to give their intensity.

As this radiation balance had previously been used primarily for relative measurements, it was carefully recalibrated so as to give absolute rates of energy dissipation. Such a calibration consists essentially in determining an average value of $d E / d T$ for the two Peltier couples, and for this purpose a completely new set of compensated calibration coils, encased in copper sheaths and with permanent potential leads, was constructed. Previous calibrations had been made with coils that had been wound on glass of such dimensions as to simulate the United Kingdom and the United States Hönigschmid primary radium standards and the Canadian national radium standard [4]. The results obtained for $d E / d T$ with the "Hönigschmid", "Canadian", and the new copper-sheathed coils are shown in table 1.

TABLE 1. Measurement of the thermoelectric power, $d E / d T$ at $25^{\circ} \mathrm{C}$

\begin{tabular}{|c|c|}
\hline Coil & $d E / d T^{\mathrm{a}}$ \\
\hline $\begin{array}{l}\text { "Hönigschmid" } \\
\text { Do } \\
\text { "Canadian“" } \\
\text { Do } \\
\text { Copper-sheathed } \\
\text { Do } \\
\text { Do } \\
\text { Do } \\
\text { Do } \\
\text { Do } \\
\text { Do } \\
\text { Do } \\
\text { Do }\end{array}$ & $\begin{array}{l}\mu w / \text { deg } \\
58.86 \\
58.95 \\
58.71 \\
58.59 \\
58.49 \\
58.67 \\
58.89 \\
58.79 \\
58.79 \\
58.60 \\
58.70 \\
58.62 \\
58.53\end{array}$ \\
\hline
\end{tabular}

a The average value of $d E / d T$ is $58.71 \mu \mathrm{w} / \mathrm{deg}$ at $25^{\circ} \mathrm{C}$

The source of polonium-210, that is illustrated in figure 1 , is such as to enable a further important check to be made in connection with the operation of the radiation balance. It has been assumed [4] that the thermal "mixing" in the gold cups is of such a magnitude as to render any differences in temperature distribution in the cups due to difference in source geometry unimportant. A series of measurements was carried out therefore with the polonium210 source, and a dummy source containing a glasswool plug and inactive silver powder, in which the energy emission from the source in the erect position, as in figure 1 , was compared with that as measured with the source and dummy upside down. In the erect position the polonium-210 is concentrated in the lower part of each cup in turn, whereas in the upside-down position a relatively good thermal insulator, the glass-wool plug, is in close proximity
TA BLE 2. Energy dissipation from polonium-210 source as measured in erect and upside-down positions

\begin{tabular}{|c|c|c|}
\hline Date, 1954 & $\begin{array}{l}\text { Energy } \\
\text { emission } \\
\text { (erect) }\end{array}$ & $\begin{array}{l}\text { Energy } \\
\text { emission } \\
\text { (upside } \\
\text { down) }\end{array}$ \\
\hline $\begin{array}{l}\text { June } 22 \\
\text { June } 29 \\
\text { July } 6 \\
\text { July } 20\end{array}$ & $\begin{array}{c}\mu w \\
1323.6 \\
1276.9 \\
1235.2 \\
1151.2\end{array}$ & $\begin{array}{c}\mu i r \\
1332.4 \\
1275.9 \\
1234.9 \\
1151.2\end{array}$ \\
\hline
\end{tabular}

TABLE 3. Energy dissipation from polonium-210 as a function of time

\begin{tabular}{|c|c|c|c|c|}
\hline Date, 1954 & $\begin{array}{l}\text { Time } \\
\text { (EST) }\end{array}$ & $\begin{array}{l}\text { Hours } \\
\text { from zero }\end{array}$ & $\begin{array}{l}\text { Observed } \\
\text { energy } \\
\text { emission }\end{array}$ & $\begin{array}{l}\text { Energy emis- } \\
\text { sion corrected } \\
\text { to zero date a } \\
\text { (using half-life } \\
\text { of } 138.39 \text { days) }\end{array}$ \\
\hline $\begin{array}{l}\text { June } 22 \\
\text { June } 29 \\
\text { July } 6 \\
\text { July } 13 \\
\text { July } 20 \\
\text { August } 24 \\
\text { August } 31 \\
\text { September } 7\end{array}$ & $\begin{array}{l}13.30 \\
13.15 \\
15.05 \\
14.40 \\
14.20 \\
13.25 \\
10.05 \\
14.30\end{array}$ & $\begin{array}{r}-167.75 \\
0 \\
169.75 \\
337.25 \\
505.25 \\
1344.25 \\
1508.75 \\
1681.25\end{array}$ & $\begin{array}{c}\mu w \\
1323.6 \\
1276.9 \\
1235.2 \\
1190.5 \\
1151.2 \\
961.43 \\
929.29 \\
899.37\end{array}$ & $\begin{array}{c}\mu w \\
1278.1 \\
1276.9 \\
1279.8 \\
1277.3 \\
1279.2 \\
1272.8 \\
1273.2 \\
1277.5\end{array}$ \\
\hline
\end{tabular}

a Average value is $1276.9 \mu \mathrm{w}$, with a standard deviation of 0.2 percent.

with the Peltier couples at the bases of the cups.

As is seen from table 2, there is no significant difference between the measurements in the two positions; the difference between the first two measurements is not outside the possible range of experimental error.

Finally, in table 3 the results are shown for rate of energy emission from the polonium-210 source on various dates of measurement. Also shown are the values that have been obtained after correction, using a half-life of 138.39 days [7], to a zero date of 13.15 hours EST on June 29, 1954, on which date, in the morning, the gamma-ray intensity was determined.

Using an alpha-particle energy of $5.301 \mathrm{Mev}$, which corresponds to a total disintegration energy of $5.404 \mathrm{Mev}$, and the average value of $1276.9 \mu \mathrm{W}$ for the energy emission, the alpha-particle activity at the above time is found to be $1,475 \times 10^{5}$ disintegrations per second. As the gamma-ray measurements were made some 3 or 4 hours earlier, it is appropriate to correct this value to $1,476 \times 10^{6}$ disintegrations per second at the time of the gamma-ray experiment.

\section{Measurement of the Gamma-Ray Intensity}

The intensity of the gamma radiation from the same polonium-210 source as used in the above experiment was determined by comparison with a cobalt-60 source, in the form of cobaltous chloride in aqueous solution, through the use of a sodiumiodide scintillation counter of high detection efficiency. The disintegration rate of the cobalt-60 source had been determined absolutely by the coincidence method. 
The experimental procedure was as follows: The polonium-210 and cobalt-60 sources of identical dimensions as in figure 1 were placed alternatively on the axis of, and $3 \mathrm{in}$. from the face of, a cylindrical sodium-iodide, thallium-activated, crystal, 5 in. in diameter and 4 in. long, as shown in figure 2. This crysta! was hermetically sealed in a thin aluminum container, using magnesium oxide as a diffuse reflector on all surfaces except that which was viewed by a 5 -in. diameter photomultiplier (type K1198). The output of the photomultiplier tube was fed through a linear amplifier into a single-channel differential pulse-height analyzer from which the intensity versus pulse-height spectra shown in figure 3 were obtained. As the sodium-iodide crystal is not quite totally absorbing, the relative efficiency for the detection of the gamma rays from the polonium and cobalt sources must be calculated. This relative efficiency is given by

$$
\epsilon=\frac{\int_{0}^{\alpha}\left(1-e^{-\mu\left(E_{1}\right) \rho x(\boldsymbol{\beta})}\right) \sin \beta d \beta}{\int_{0}^{\alpha}\left(1-e^{-\mu\left(E_{2}\right) \rho x(\boldsymbol{\beta})}\right) \sin \beta d \beta},
$$

where

$$
\begin{aligned}
x(\beta) & =t \sec \beta \quad 0 \leq \beta \leq \tan ^{-1} r /(D+t)=\beta^{\prime} \\
x(\beta) & =r \csc \beta-D \sec \beta \quad \beta^{\prime} \leq \beta \leq \tan ^{-1} r / D=\alpha \\
\mu(E) & =\text { mass absorption coefficient for energy } E \\
\rho & =\text { density. }
\end{aligned}
$$

The values of $\mu(E)$ for sodium iodide were obtained from the tables of White [8] for energies corresponding to those of 0.804 and $1.25 \mathrm{Mev}$, the latter value being the average energy of the cobalt-60 gamma rays; this artifice introduces negligible error. For the geometry and dimensions given in figure 3 , one obtains a value 1.123 for the relative detection efficiency, $\epsilon$, by numerical integration of eq (1). The disintegration rate for the polonium gamma ray is given by

$$
N_{0 \gamma}\left(\mathrm{Po}^{210}\right)=\frac{A\left(\mathrm{Po}^{210}\right)}{\epsilon A\left(\mathrm{Co}^{60}\right)} N_{0}\left(\mathrm{Co}^{60}\right)
$$

where $A$ is the area under each of the spectra of figure 3 . The area under the cobalt-60 spectrum is given proper weight to take into account the cascade nature of the gamma radiation. Four determinations of the relative areas give a value $A\left(\mathrm{Po}^{210}\right) / A\left(\mathrm{Co}^{60}\right)=0.648$, thus we obtain a value for the disintegration rate of the polonium gamma ray in terms of the $\mathrm{Co}^{60}$ disintegration rate, i. e.,

$$
N_{0 \gamma}\left(\mathrm{Po}^{210}\right)=0.5770 N_{0}\left(\mathrm{Co}^{60}\right) .
$$

The estimated error of this relationship may be as great as 5 percent because of the uncertainty in correcting for secondary effects, such as backscattering into the detector.

The disintegration rate of the cobalt-60 source was determined by the gamma-gamma coincidence method. This disintegration rate in terms of ob-

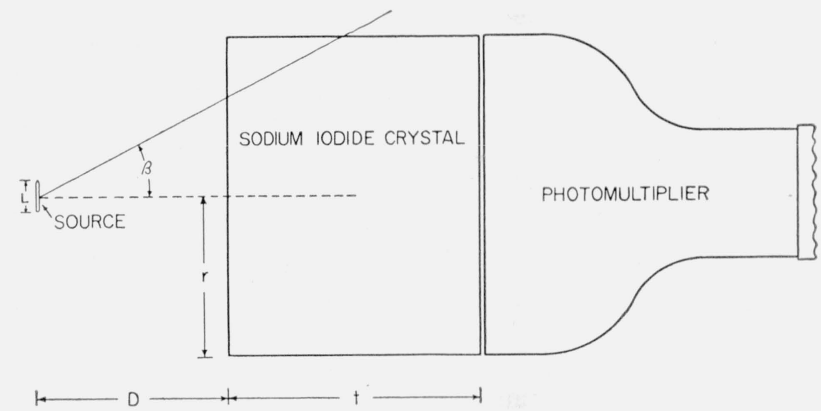

FIGURE 2. Geometrical arrangement for comparison of gammaray spectra of cobalt-60 and polonium-210 sources.


Figure 3. Scintillation pulse-height spectra of (a) polonium-210 source, (b) cobalt-60 source. 
servable quantities is given by

$$
N_{0}=\frac{N_{1} N_{2}}{N_{c}} \frac{\zeta_{1}+\zeta_{2}}{\left(\zeta_{1}+1\right)\left(\zeta_{2}+1\right)} f(\vartheta)
$$

where $N_{1}$ and $N_{2}$ are the counting rates in each of two similar counters, $N_{c}$ is the number of coincidence counts between the two counters, $\zeta_{1}$ and $\zeta_{2}$ are the ratio of efficiencies for the two gamma rays in the first and second counters, respectively, and $f(\vartheta)$ is a function depending on the geometry of the source, counters, and any angular correlation between the coincident gamma rays. For the cobalt-60 source where there is a definite angular correlation, this function takes the form (9).

$$
\begin{aligned}
f(\vartheta)=1 & +\left(J_{2} / J_{0}\right)^{2} 0.1020 P_{2}(\cos \vartheta) \\
& +\left(J_{4} / J_{0}\right)^{2} 0.0091 P_{4}(\cos \vartheta) \\
& +\frac{1}{4}(L / D)^{2}\left[1-P_{1}(\cos \vartheta)\right]\left\{0.1020 P_{1}(\cos \vartheta)\right. \\
& \left.+0.0091\left[P_{1} \cos \vartheta+\frac{7}{3} P_{3}(\cos \vartheta)\right]\right\}
\end{aligned}
$$

The terms containing $J_{l}$ are attenuation coefficients due to the finite angular resolution of the counters, where the $J_{l}$ is given by

$$
J_{l}(\alpha)=\int_{0}^{\alpha} P_{l}(\cos \beta)\left(1-e^{-\mu(E) \rho x(\beta)}\right) \sin \beta d \beta .
$$

The expression for $x(\beta)$ is of the same form as in eq (1). The last term in eq (4) is a correction term [10] due to the finite length of the source. The numerical coefficients of the Legendre polynomials are theoretical values for a cascade transition between states with spins $4 \rightarrow 2 \rightarrow 0$ [11].

With a geometry as shown in figure 4 , the theoretically expected anisotropy is $\mathrm{f}(\boldsymbol{\pi}) / \mathrm{f}(\boldsymbol{\pi} / 2)=1.150$. The experimentally observed value was 1.147 , indicating that when the corrections for the finite sizes of source and counters are accounted for, the measured differential anisotropy of 1.164 is well within the probable error (approximately 1 percent) of being the same as the theoretically expected value of 1.167 [11].

Several determinations of the disintegration rate under different geometrical conditions gave a value

$$
N_{0}\left(\mathrm{Co}^{60}\right)=(3.087 \pm 0.030) \times 10^{4} \mathrm{dps} .
$$

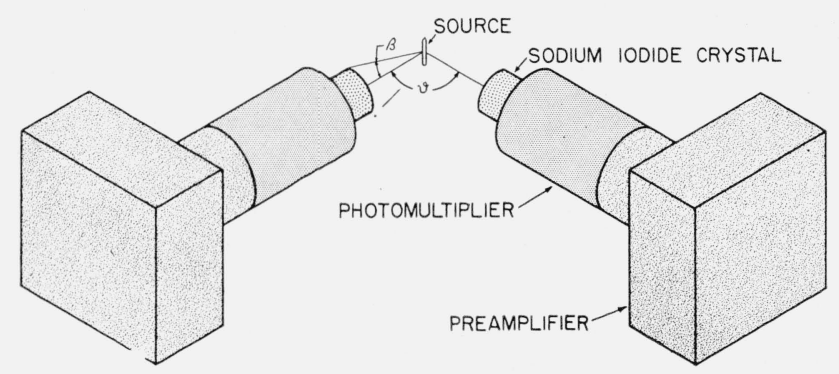

Figure 4. Geometrical arrangement for coincidence measurements on cobalt-60 source.
Using this value, we obtain as the intensity of the polonium-210 gamma rays

$$
N_{0 \gamma}\left(\mathrm{Po}^{210}\right)=(1.782 \pm 5 \%) \times 10^{4} \mathrm{dps} .
$$

\section{Branching Ratio}

Combining the gamma-ray and alpha-particle intensities, the following value for their ratio is obtained:

$$
\frac{\Lambda_{0} \gamma}{N_{0} \alpha}=\frac{1.782 \times 10^{4}}{1.476 \times 10^{9}}
$$

$=\left(1.20_{7} \pm 0.06_{0}\right) \times 10^{-5}$ gamma quantum per alpha particle.

This value of $(1.21 \pm 0.06) \times 10^{-5}$ is to be compared with that of $(1.8 \pm 0.14) \times 10^{-5}$ obtained by Grace, Allen, West, and Halban [1] and $(1.6 \pm 0.2) \times 10^{-5}$ by M. Riou [12]. The internal conversion of $\mathrm{K}$-shell electrons in polonium-210 is $0.0084[13$ and correction for L-shell conversion electrons [14] gives a total conversion coefficient of 0.0107 . Using this value, the branching ratio is found to be $1.22 \times$ $10^{-5} \pm 5$ percent.

Grateful acknowledgment is made to A. Schwebel for his assistance in preparing the polonium-210 source from the solution received, to H. B. Kearney and M. Pavey of the Canadian Radium and Uranium Corporation for their cooperation in the preparation of the purified polonium-210 solution, and to $\mathrm{H}$. W. Koch for the use of the large sodium-iodide crystal.

\section{References}

[1] M. A. Grace, R. A. Allen, D. West, and H. Halban, Proc. Phys. Soc. 64, 493 (1951).

[2] R. W. Pringle, H. W. Taylor, and S. Standil, Phys. Rev. 87, 384 (1952).

[3] H. Bradt, P. C. Gugelot, O. Huber, H. Medicus, P. Preiswerk, and P. Scherrer, Helv. Phys. Acta 19, 77 (1946).

[4] W. B. Mann, J. Research NBS 53, 277 (1954) RP2545.

[5] E. R. Collins, C. D. McKenzie, and C. A. Ramm, Nature 16\%, 682 (1951).

[6] W. J. Sturm and V. Johnson, Phys. Rev. 83, 542 (1951).

[7] D. C. Ginnings, Anne F. Ball, and D. T. Vier, J. Research N BS 50, 75 (1953) RP2392.

[8] G. R. White NBS report 1003 (unpublished).

[9] M. E. Rose, Phys. Rev. 91, 610 (1953).

[10] J. S. Lawson, Jr., and H. Frauenfelder, Phys. Rev. 91, 649 (1953).

[11] S. P. Lloyd, Phys. Rev. 83, 716 (1951).

[12] M. Kiou, J. phys, radium 13, 244 (1952)

[13] M. E. Rose, G. H. Goertzel, and C. L. Perry, K-shell internal conversion coefficients, ORNL Report No. 1023 (1951).

[14] D. E. Alburger and G. Friedlander, Phys. Rev. 81, 523 (19.51).

Washington, September 22, 1954. 